\title{
Universiteit
}

Leiden

The Netherlands

\section{Phase coherence, visibility, and the superfluid-mott-insulator transition on one-dimensional optical lattices}

Sengupta, P.; Rigol, M.; Batrouni, G.G.; Denteneer, P.J.H.; Scalettar, R.T.

\section{Citation}

Sengupta, P., Rigol, M., Batrouni, G. G., Denteneer, P. J. H., \& Scalettar, R. T. (2005). Phase coherence, visibility, and the superfluid-mott-insulator transition on one-dimensional optical lattices. Physical Review Letters, 95(22), 220402. doi:10.1103/PhysRevLett.95.220402

Version: $\quad$ Not Applicable (or Unknown)

License: $\quad$ Leiden University Non-exclusive license

Downloaded from: https://hdl.handle.net/1887/71385

Note: To cite this publication please use the final published version (if applicable). 


\title{
Phase Coherence, Visibility, and the Superfluid-Mott-Insulator Transition on One-Dimensional Optical Lattices
}

\author{
P. Sengupta, ${ }^{1}$ M. Rigol, ${ }^{2}$ G. G. Batrouni, ${ }^{3}$ P. J. H. Denteneer,${ }^{4}$ and R. T. Scalettar ${ }^{2}$ \\ ${ }^{1}$ Department of Physics, University of California, Riverside, California 92521, USA \\ ${ }^{2}$ Physics Department, University of California, Davis, California 95616, USA \\ ${ }^{3}$ Institut Non-Linéaire de Nice, UMR 6618 CNRS, Université de Nice-Sophia Antipolis, \\ 1361 route des Lucioles, 06560 Valbonne, France \\ ${ }^{4}$ Lorentz Institute, Leiden University, P.O. Box 9506, 2300 RA Leiden, The Netherlands
}

(Received 3 August 2005; published 22 November 2005)

\begin{abstract}
We study the phase coherence and visibility of trapped atomic condensates on one-dimensional optical lattices, by means of quantum Monte Carlo simulations. We obtain structures in the visibility similar to the kinks recently observed experimentally by Gerbier et al. [Phys. Rev. Lett. 95, 050404 (2005); cond-mat/ 0507087]. We examine these features in detail and offer a connection to the evolution of the density profiles as the depth of the lattice is increased. Our simulations reveal that, as the interaction strength $U$ is increased, the evolution of superfluid and Mott-insulating domains stall for finite intervals of $U$. The density profiles do not change with increasing $U$. We show here that in one dimension the visibility provides unequivocal signatures of the melting of Mott domains with densities larger than 1 .
\end{abstract}

DOI: 10.1103/PhysRevLett.95.220402

PACS numbers: 03.75.Hh, 03.75.Lm, 05.30.Jp

The realization of trapped Bose-Einstein condensates in ultracold atoms on optical lattices has opened up the possibility of observing experimentally various quantum phases-e.g., superfluid (SF) and Mott-insulator (MI) and the study of the nature of the transitions between them in a well-controlled manner. Indeed, the existence of SF and MI phases on optical lattices was established experimentally $[1,2]$, where it was demonstrated that, by increasing the optical lattice depth, the system passes from a SF phase to a predominantly MI one. Contrary to the unconfined case, in traps SF and MI domains coexist, in general. Hence, the passage from SF to MI has to be understood as a crossover rather than as a quantum phase transition [3,4], although a vestige of the latter remains in the guise of local quantum criticality $[5,6]$.

The experimental systems can be modeled by the boson Hubbard model [7], described in one dimension (1D) by

$$
\begin{aligned}
H= & -t \sum_{i}\left(a_{i}^{\dagger} a_{i+1}+a_{i+1}^{\dagger} a_{i}\right)+\mu \sum_{i} n_{i}+V_{T} \sum_{i} x_{i}^{2} n_{i} \\
& +U / 2 \sum_{i} n_{i}\left(n_{i}-1\right),
\end{aligned}
$$

where $L$ is the number of sites and $x_{i}=i a$ is the coordinate of the $i$ th site, and $a$ is the lattice constant. The hopping parameter $t$ sets the energy scale, $n_{i}=a_{i}^{\dagger} a_{i}$ is the number operator, and $\left[a_{i}, a_{j}^{\dagger}\right]=\delta_{i j}$ are bosonic creation and destruction operators. $V_{T}$ is the curvature of the trap, while the repulsive contact interaction is given by $U$. The chemical potential $\mu$ controls the number of particles. The phase diagram of this model in the absence of the confining trap has been extensively studied with the goal of elucidating the various quantum phases it exhibits [8-10] and the transitions between them.
The key experimental signature of these phases lies in the interference pattern observed after the release of the gas from the trap and subsequent free expansion - an SF (MI) produces a sharp (diffuse) interference pattern reflecting the presence (loss) of phase coherence. Phase coherence, especially in reduced dimensionality, continues to be of great interest both experimentally and theoretically. Particular attention has been focused recently on mechanisms which can destroy quasilong-range coherence in systems on optical lattices, especially in 1D [2]. Our focus in this Letter is the role, in $1 \mathrm{D}$, of the passage from the SF to the MI phase in destroying phase coherence, which can be studied in matter wave interference.

Whereas previous studies of the SF-MI transition focused on the height [1] and width [2] of the central interference peak, an alternative scheme was proposed recently [11] where the reduction of phase coherence approaching the MI was characterized by the visibility of interference fringes,

$$
\mathcal{V}=\frac{S_{\max }-S_{\min }}{S_{\max }+S_{\min }}
$$

Here $S_{\max }$ and $S_{\min }$ are the maximum and minimum values of the momentum distribution function,

$$
S(\mathbf{k})=\frac{1}{L} \sum_{j, l} e^{i \mathbf{k} \cdot\left(\mathbf{r}_{\mathbf{j}}-\mathbf{r}_{l}\right)}\left\langle a_{j}^{\dagger} a_{l}\right\rangle .
$$

It was observed that, as the optical lattice depth [equivalent to the Hubbard $U / t$ in Eq. (1)] is increased, the visibility decreases until special values of $U$ are reached where $\mathcal{V}$ displays "kinks," after which it decreases again. It was also shown [11] that the values of $U$ at which such kinks are observed are reproducible and that they depend on the 
filling (number of atoms). A perturbative treatment of the homogeneous MI phase [11] has shown that $\mathcal{V}$ decreases as $U^{-1}$, improving on previous numerical results on small systems [12].

Gerbier et al. [11] proposed that the kinks are linked to a redistribution of the density as the SF shells transform into MI regions with several atoms per site. In this Letter, we examine in detail the presence and properties of these visibility kinks with the help of quantum Monte Carlo simulations of the boson Hubbard model using the stochastic series expansion method [13]. We focus on 1D optical lattices and show that, while the kinks are indeed related to the redistribution in the density associated with SF-MI transition, they are not produced solely by the transformation of SF shells into MI domains. Indeed, we find $\mathcal{V}$ reveals other subtle details of density redistributions with $U$.

We start our study with the simplest case, i.e., a system in which the density in the middle of the trap never reaches $n=2$, so that when the interaction strength is increased, only Mott domains with $n=1$ appear. In Fig. 1(a), we show the visibility and $S_{\max }$ as functions of $U / t$. As in the experiments [11], $\mathcal{V}$ decreases with increasing $U / t$-reflecting the decrease of $S_{\max }$ and the increase of $S_{\min }$ (not shown in the figure) - with an intermediate region, over which it remains fairly constant. Two kinks can be observed in both $\mathcal{V}$ and $S_{\max }$. The first one (less evident) occurs around $U / t=6.1$, and the second one around $U / t=7.0$.
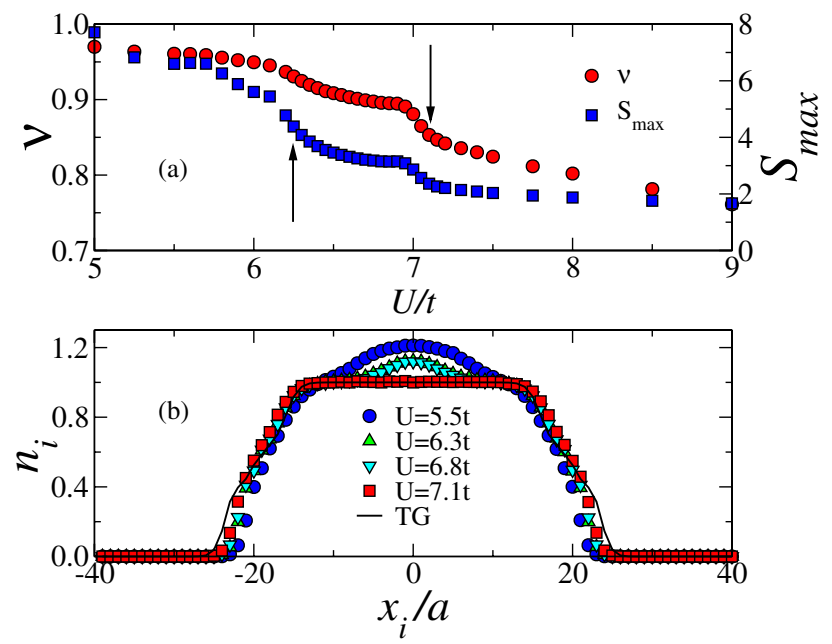

FIG. 1 (color online). (a) Visibility $\mathcal{V}$ and $S_{\max }$ as functions of the on-site interaction $U / t$. Initially, $\mathcal{V}$ decreases as $U / t$ increases. After $U / t \approx 6.3$, its rate of reduction decreases due to the freezing of the density profiles (see text). The fast decrease after $U / t \approx 7$ is related to the formation of the central Mott core. (b) Density profiles at four different values of $U / t$ and in the TG regime. The profiles for $U / t=6.3$ and 6.8 virtually coincide. The system under consideration has 40 bosons on an 80 -site chain and a trapping potential $V_{T} a^{2}=0.01 t$. Error bars on the data in this and all subsequent figures are smaller than the symbol sizes.
Density profiles corresponding to four values of $U / t$ are depicted in Fig. 1(b). The density profile for $U / t=6.3$ in Fig. 1(b) shows that the first kink in Fig. 1(a) (signaled by the first arrow) is related to the emergence of two MI plateaus at the sides $\left[x_{i} / a \approx \pm(8-12)\right]$ of a central SF region. The second kink in Fig. 1(a) is related to the formation of a full MI domain in the middle of the trap, which produces more evident structures in $\mathcal{V}$ and $S_{\max }$. This occurs for $U / t=7.1$ as shown in Fig. 1(b) and signaled by the second arrow in Fig. 1(a). Plotting $\mathcal{V}$ and $S_{\max }$ as a function of $U / t$ allows us to present more precisely the position and shape of the kinks: In experiments, the control parameter is the ratio between the lattice depth and the recoil energy, which produces exponential changes in $U / t[7,11]$.

One unexpected feature is the freezing of the density profiles before the full MI forms in the middle of the trap, which coincides with the plateaulike behavior of $\mathcal{V}$ and $S_{\max }$ between the two arrows. As $U / t$ is increased between 6.3 and 6.8, almost no changes occur in the density distribution; i.e., the bosons are no longer being pushed out of the central regions to the outlying zones, even though $U / t$ continues to increase. This behavior may seem surprising, as the central region of the system is SF, i.e., compressible, but can be explained by the presence of the emerging MI domains at the sides. The central SF region gets trapped between them, and the interaction $U / t$ first has to increase a finite amount before particles can be transferred to the SF regions at the edge against the substantially larger trap energy there.

This can be better understood by computing the total trapping $\left(E_{T}\right)$ and interaction $\left(E_{P}\right)$ energies as functions of $U / t$ [Fig. 2(a)]. In the interval $U / t=6.3-6.8$, both quantities exhibit a plateau, which is also reflected in the chemical potential of the system [Fig. 2(b)]. This occurs even though the total energy (not shown) increases con-

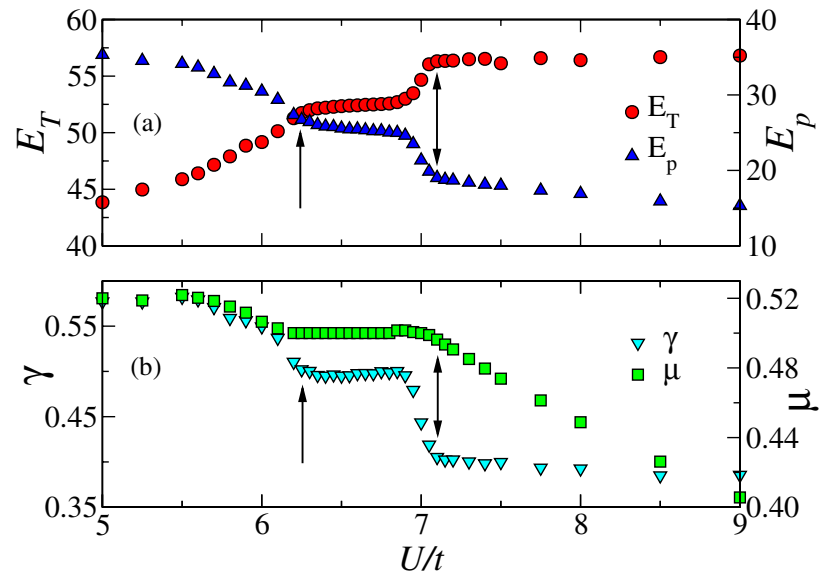

FIG. 2 (color online). (a) Trapping $\left(E_{T}\right)$ and interaction $\left(E_{P}\right)$ energies as functions of $U / t$. (b) Ratio $\gamma=\left|E_{p} / E_{k}\right|$ of potential to kinetic energy, and the chemical potential $(\mu)$ needed to maintain $N_{b}=40$. The results are for the system in Fig. 1 . 
tinuously, due to the continuous decrease in magnitude of the (negative) kinetic energy $\left(E_{K}\right)$ of the bosons. One can then see that the formation of the full MI plateau is accompanied by a fast increase in the total trap energy of the system by $\sim 4 t$, the bandwidth in $1 \mathrm{D}$. On the other hand, the decrease of interaction energy produced by the formation of the MI plateau is even larger, $\sim 6 t$. Thus, in experiments, abrupt changes can occur in the density profiles even if the lattice depth is increased slowly. This can produce the escape of particles from the trap, heating, or other unexpected features.

In Fig. 2(b), we also show $\gamma=\left|E_{P} / E_{K}\right|$, the ratio of potential to kinetic energy. This quantity is different from the one often used to characterize trapped bosons on lattices $\gamma_{L}=U / t$ [14]. In contrast to $\gamma_{L}$, for the system in Figs. 1 and 2, $\gamma$ decreases with increasing $U$. This occurs because the density all over the trap becomes $n \leq 1$, and the double occupancy is strongly suppressed. [In the Tonks-Girardeau limit (TG), i.e., $U \rightarrow \infty, \gamma=0$, while $\gamma_{L}=\infty$.] Like the visibility and the chemical potential, $\gamma$ remains almost unchanged in the region where the density profiles are frozen.

As the on-site interaction is further increased, no more abrupt changes occur in the trap. The density profile remains almost the same, as seen in Fig. 1(b), where we have also plotted the exact result in the TG limit. The visibility and $S_{\max }$ reduce continuously to $\mathcal{V}_{\mathrm{TG}}=0.39$ and $S_{\max }^{\mathrm{TG}}=$ 1.3 (obtained using the approach presented in Ref. [15]). Notice that even when $U \rightarrow \infty$ the visibility does not vanish, due to SF domains surrounding the MI.

When the density at the center of the trap is higher, and exceeds 2, the evolution of the visibility with the on-site repulsion exhibits an even richer structure. Results for a system in that regime are presented in Fig. 3(a). The visibility, up to $U / t \sim 13$, is very similar to Fig. 1(a). Density profiles for three values of $U$ in that interval are presented in Fig. 4(a). One can see that the emergence of MI regions with $n=1$, and $n=2$ surrounding SF regions with $2>n>1$, and $n>2$, respectively, produces a plateau in $\mathcal{V}$ due to a freezing of the density profiles when increasing $U$. In Fig. 3(a), the formation of the $n=2$ plateau abruptly reduces the visibility, similar to the formation of the $n=1$ plateau in Fig. 1(a).

However, the behavior above $U / t=13$ has additional structures compared to Fig. 1(a). In order to understand the origin of these visibility features, we have plotted in Fig. 3(b) the integrated density over 20 lattice sites around the center of the trap $N_{c}=\sum_{i=-10}^{10} n_{i}$. A clear one to one mapping between the features in the visibility and plateau in $N_{c}$ is seen. The visibility kinks result not from the formation of new SF or MI regions but rather from a redistribution of bosons between the MI states with $n=$ 2 and $n=1$. As seen in Figs. 3(b) and 4, such a redistribution occurs discontinuously in $U$. In addition, since the SF domains with $2>n>1$ can increase their sizes during
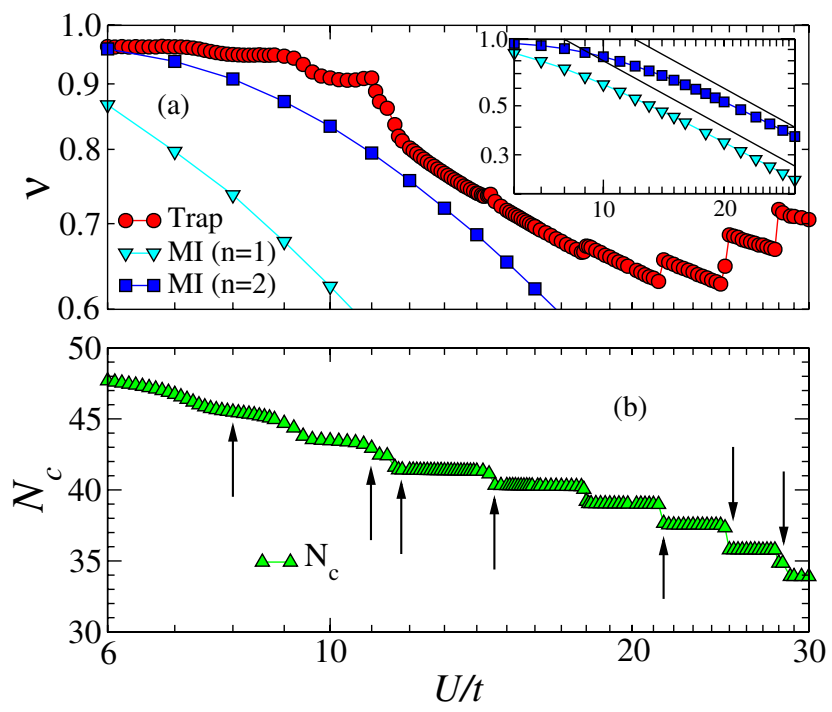

FIG. 3 (color online). (a) Visibility $\mathcal{V}$ as a function of the onsite interaction $U / t$, for $N_{b}=60$ and $V_{T} a^{2}=0.06 t$, parameters which allow both $n=1$ and $n=2$ Mott regions to exist. For comparison, results for pure Mott-insulating phases with $n=1$ and $n=2$ in open lattices without a trap are also given. In the inset, the straight lines show the perturbative results of Ref. [11] in 1D (see text). (b) Integrated density over 20 lattice sites around the center of the trap.

such a process, the visibility can increase [see, for example, the kinks around $U / t=14.6$ and 21.5 in Fig. 3(a) and the corresponding density profiles in Fig. 4].

The above features are not restricted to the 1D character of the system and could be observed in higher dimensions. However, as $U$ is increased even further $(U \gtrsim 25 t)$, a purely $1 \mathrm{D}$ effect sets in. As the MI plateau with $n=2$ melts, correlations start to develop between the two disconnected SF domains with $2>n>1$. This produces a large increase of the visibility, as seen in Fig. 3(a). [The corresponding density profiles are shown in Fig. 4(b).] In $1 \mathrm{D}$, this increase in the visibility provides an unambiguous signature of the presence, and melting, of the $n=2$ (or larger) MI domain. This can be useful for understanding the dynamics of strongly correlated bosons in 1D [16].

For very large values of $U$, beyond the ones in Fig. 3(a), the $2>n>1$ SF domain will eventually disappear, as

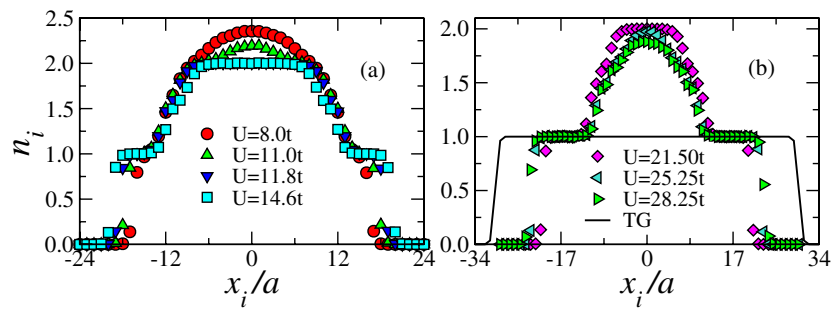

FIG. 4 (color online). Density profiles corresponding to the points signaled by arrows in Figs. 3 and 5. The continuous line in (b) is the result in the TG regime. $N_{b}=60$ and $V_{T} a^{2}=0.06 t$. 
occurs in Fig. 1(a), producing a further reduction in the visibility. In the TG regime, we obtain (for these parameters) $\mathcal{V}_{\mathrm{TG}}=0.02$. The corresponding density profile can be also seen in Fig. 4(b).

We have also plotted in Fig. 3(a) the values obtained for the visibility in homogeneous systems with 60 bosons and densities $n=1$ and $n=2$. (We have used open boundary conditions as they are closer to the trapped case.) These results in homogeneous systems are very different from the ones in the trapped case. Because of the existence of SF domains, the visibility in the trap is always larger than that in the homogeneous case. In the region of interest, where the MI plateau emerges, and melts, no extrapolation is possible from the uniform case. Only for very large values of $U$, after a MI domain appears in the center of the trap, can one can expect the uniform and trapped systems to behave similarly. In the inset, we have compared the results for the homogeneous systems with those obtained in Ref. [11] [ $\left.\mathcal{V}_{1 \mathrm{D}}=4(n+1) t / U\right]$. For the largest values of $U$, one can see that the $t / U$ power law starts to develop, but its prefactor is still different from $4(n+1)$, so that very large values of $U$ are needed for a good agreement in 1D.

We conclude by showing in Fig. 5 the behavior of $\gamma$ in the system of Figs. 3 and 4. In this case, since the density at the middle of the trap is larger than 1, i.e., there is significant double occupancy in this region, $\gamma\left(\right.$ and $E_{P}$ ) increases with $U / t$. It also exhibits the same jumps produced by the redistribution of particles in Fig. 4. As in the system in Figs. 1 and 2, this occurs even when the total energy of the system $\left(E_{S}\right)$ increases continuously with $U$, as can also be seen in Fig. 5.

In this Letter, we have explored the evolution of the visibility of trapped atomic gases in one-dimensional optical lattices using quantum Monte Carlo simulations. We have shown that the visibility behaves very similarly to that observed experimentally. In particular, it has kinks associated with redistribution of density amongst Mott-insulating and superfluid regions within the trap. In addition, we have also exhibited several other novel features of the visibility evolution in 1D, such as a large increase due to the melting, with increasing $U / t$, of $n>1$ MI plateaus. We have demonstrated that the evolution of the density distribution with interaction strength exhibits pauses. That is, at certain values of $U$ the density distribution, and other observables, do not change even when the interaction strength increases over a range as large as $t / 2$. We have shown that the emergence of this static behavior is associated with the formation of Mott-insulating plateaus away from the trap center. These plateaus block the transfer of bosons to the outer parts of the system and, hence, cause the evolution to stall. While many quantities in trapped Bose systems are well described by the local density approximation, it is not clear that approach will capture the above behavior, in-

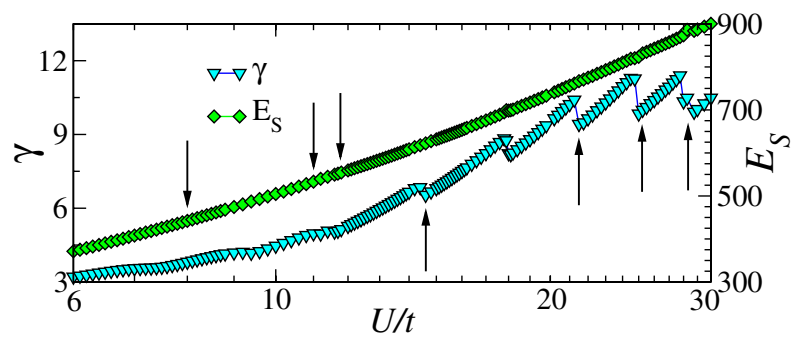

FIG. 5 (color online). Ratio $\gamma=\left|E_{p} / E_{k}\right|$ of potential to kinetic energy, and the total energy of the system $\left(E_{S}\right)$ vs $U / t$, for the system in Figs. 3 and 4. $N_{b}=60$ and $V_{T} a^{2}=0.06 t$.

cluding the kinks in the visibility. This is because these effects are intrinsically tied to the competition of the trap versus kinetic and interaction energies in systems where the SF and MI domains are of finite width, as in the ones explored in recent experiments.

We thank I. Bloch and M. Troyer for helpful discussions. M. R. and R. T. S. were supported by NSF-DMR-0312261, P. J.H. D. by Stichting FOM.

[1] M. Greiner et al., Nature (London) 415, 39 (2002).

[2] T. Stöferle et al., Phys. Rev. Lett. 92, 130403 (2004).

[3] G. G. Batrouni et al., Phys. Rev. Lett. 89, 117203 (2002).

[4] S. Wessel, F. Alet, M. Troyer, and G. G. Batrouni, Phys. Rev. A 70, 053615 (2004).

[5] M. Rigol, A. Muramatsu, G. G. Batrouni, and R.T. Scalettar, Phys. Rev. Lett. 91, 130403 (2003).

[6] G. G. Batrouni, F. F. Assaad, R. T. Scalettar, and P. J. H. Denteneer, Phys. Rev. A 72, 031601(R) (2005).

[7] D. Jaksch et al., Phys. Rev. Lett. 81, 3108 (1998).

[8] M.P. A. Fisher, P. B. Weichman, G. Grinstein, and D. S. Fisher, Phys. Rev. B 40, 546 (1989).

[9] G. G. Batrouni, R. T. Scalettar, and G. T. Zimanyi, Phys. Rev. Lett. 65, 1765 (1990).

[10] K. G. Singh and D. S. Rokhsar, Phys. Rev. B 46, 3002 (1992); N. V. Prokof'ev and B. V. Svistunov, Phys. Rev. Lett. 80, 4355 (1998); T.D. Kuhner, S. R. White, and H. Monien, Phys. Rev. B 61, 12474 (2000).

[11] F. Gerbier et al., Phys. Rev. Lett. 95, 050404 (2005); Phys. Rev. A 72, 053606 (2005).

[12] R. Roth and K. Burnett, Phys. Rev. A 67, 031602(R) (2003).

[13] A. W. Sandvik, J. Phys. A 25, 3667 (1992); Phys. Rev. B 59, R14 157 (1999).

[14] M. A. Cazalilla, Phys. Rev. A 70, 041604(R) (2004).

[15] M. Rigol and A. Muramatsu, Phys. Rev. A 70, 031603(R) (2004); 72, 013604 (2005).

[16] C. D. Fertig et al., Phys. Rev. Lett. 94, 120403 (2005); M. Rigol et al., Phys. Rev. Lett. 95, 110402 (2005); A. M. Rey et al., Phys. Rev. A 72, 033616 (2005); G. Pupillo et al., cond-mat/0505325. 